\title{
Nordiques
}

39 | 2020

Varia

\section{Andreas Malm, Comment saboter un pipeline}

La fabrique 2020

Thierry Maricourt

\section{(2) OpenEdition \\ Journals}

Édition électronique

URL : http://journals.openedition.org/nordiques/614

DOI : $10.4000 /$ nordiques.614

ISSN : 2777-8479

Éditeur :

Association Norden, Bibliothèque de Caen la mer

Référence électronique

Thierry Maricourt, «Andreas Malm, Comment saboter un pipeline », Nordiques [En ligne], 39 | 2020, mis en ligne le 01 novembre 2020, consulté le 13 mars 2021. URL : http://journals.openedition.org/ nordiques/614; DOI : https://doi.org/10.4000/nordiques.614

Ce document a été généré automatiquement le 13 mars 2021

Nordiques 


\section{Andreas Malm, Comment saboter un pipeline}

La fabrique 2020

Thierry Maricourt

\section{RÉFÉRENCE}

Andreas Malm, Comment saboter un pipeline (How to blow up a pipeline, 2020), trad. de l'anglais Étienne Dobenesque, La Fabrique, 2020

1 À la différence des nombreux combats s'étalant sur le dernier siècle qu'Andreas Malm (né en 1977 et auteur d'un autre essai, L'Anthropocène contre l'histoire, La Fabrique, 2017) cite dans Comment saboter un pipeline et qui ont posé la question, à un moment ou à un autre, de l'emploi stratégique de la violence ou de la non-violence (apartheid en Afrique du Sud, droits des Afro-Américains aux USA, départs des colons britanniques de l'Inde, etc.), il est difficile, aujourd'hui, de nommer un responsable unique dans la lutte contre le réchauffement climatique. Cela explique sans doute l'absence de véritable succès du mouvement écologiste et ce, malgré les innombrables et puissantes manifestations de ces récentes années - puisque nous serions tous coupables, car tous consommateurs, serait-ce à notre corps défendant, d'une énergie issue en grande partie de sources non renouvelables, contre qui s'élever? Sinon l'affreux capitalisme, source de tous nos maux - comme on le sait-, mais ce n'est même pas certain, puisque des États prétendument communistes n'ont pas hésité à ravager leur territoire et celui d'autres pays au nom du productivisme industriel et de la consommation (URSS et bloc de l'Est, hier, Chine, de nos jours, etc.). Plus que le capitalisme, c'est donc l'industrialisation du monde, garante annoncée d'un mieux-être généralisé (production de masse de biens de consommation courante, du grille-pain à la tondeuse autotractée, du smartphone à l'appartement climatisé), qui est cause du désastre en cours. Industrialisation et capitalisme sont liés, certes, et à propos des pays dits communistes, on a pu parler avec raison de capitalisme d'État. États capitalistes et États dits communistes, tous de vouer 
le même culte à la productivité - le consumérisme, cette propension à consommer, selon le dictionnaire, avec l'idée de consumer, c'est-à-dire de s'adonner à ce qui se résume à une politique de la terre brûlée.

Il nous semble ainsi qu'il manque une dimension dans le passionnant essai d'Andreas Malm, maître de conférences en géographie humaine en Suède et militant pour le climat: le pourquoi de la constante opposition meurtrière entre l'être humain et la nature. Ce contexte qui à l'origine est le sien, qui est peut-être toujours le sien, et qu'il n'a cesse de modeler et de remodeler jusqu'à le détruire. Hormis les peintures rupestres, les plus anciennes traces que l'homme a laissées de son passage au cours de son histoire-préhistoire sont des blessures infligées à la nature : surfaces gigantesques déboisées, paysages arasés, villes écartelées, construction de bâtiments et de monuments démesurés (pyramides, églises, mosquées, temples, basiliques, etc., et autres édifices mégalomaniaques), ouvrages d'art (ponts, barrages...) ayant bouleversé des écosystèmes entiers, routes à foison... (Le maillage routier de plus en plus dense atteint aujourd'hui les zones les plus reculées de la planète et contribue grandement à l'extinction des espèces.) Citons également les sites industriels, les centrales nucléaires, l'extension urbaine démentielle, etc. Ce mépris manifesté par l'homme pour la faune et la flore ne remonte pas à une époque déterminée, il se retrouve dans les âges les plus anciens, dès les tout premiers temps de l'agriculture. ${ }^{1} \mathrm{Et}$ ce mépris explique peut-être pourquoi la nature n'est, n'a jamais été et ne sera jamais (sauf rupture radicale future dans l'intellect) considérée que comme un terrain de jeu pour les êtres humains, et non comme une composante essentielle de son développement.

Dès lors, la problématique au centre de ce livre, Comment saboter un pipeline (sans point d'interrogation) - convient-il de préconiser, ou non, la violence pour s'opposer à l'usage des combustibles d'origine fossile -, paraît presque secondaire. Oui, bien sûr, pourrait-on répondre, à l'instar de l'auteur, puisque la violence, tout comme la nonviolence, ne sauraient être des valeurs en soi, mais uniquement des éléments stratégiques d'un affrontement général. Hitler plus puissant que jamais, fallait-il réclamer « la paix à tout prix » (comme si y exercèrent le philosophe Félicien Challaye, qui deviendra un partisan du régime de Vichy, et un certain nombre de pacifistes pour lesquels mieux valait «la paix désarmée face à Hitler » que la guerre) et se retrouver désarmé totalement, moralement parlant? Autrement dit, désarmé, au premier degré, face à des hommes pour lesquels la violence n'était pas un souci - plutôt une façon de régner en instaurant la peur absolue - donc quelque chose de positif, selon eux, eu égard à l'objectif, ce que les pacifistes ont toujours eu du mal à comprendre.

$\mathrm{Si}$, la réponse, Andreas Malm la donne dès les premières pages de son livre, il compare ensuite, estimons-nous, des situations qui ne sont pas vraiment comparables. Alors que les publications sur les questions écologiques fleurissent, elles omettent très souvent la question fondamentale du pouvoir (la catastrophe climatique en cours est pourtant le résultat d'un pouvoir exercé par une classe sociale, celle des nantis, tant d'études le démontrent, notons en France les travaux, parmi d'autres, du couple Michel PinçonMonique Pinçon Charlot), comme si chaque individu détenait la même part de responsabilité et accusant le conducteur d'une vieille voiture diesel au même titre que le directeur de l'usine automobile ou les actionnaires - qui eux ont fait le choix du diesel par souci de rentabilité, c'est-à-dire d'accroissement des gains. Songeons, ce n'est qu'un exemple, au Mouvement Colibris et à tous ces auteurs de livres gentillets qui nous vantent l'adéquation entre la préservation de la nature et le bien-être individuel. 
Et la lutte des classes? Définitivement reléguée dans les poubelles de l'histoire? Malm, lui, essaie de mettre les points sur les « $\mathrm{i}$ », et c'est tant mieux. « Il y a une corrélation très étroite entre le revenu et la richesse d'un côté et les émissions de $\mathrm{CO} 2$ de l'autre. » Il ajoute: "Il ne fait aucun doute que les classes dirigeantes sont foncièrement incapables de répondre à la catastrophe autrement qu'en la précipitant. »

Les travaux du Français Jacques Sémelin sur la "résistance civile", ces différentes façons de répondre à l'oppression, même s'ils datent quelque peu pour certains et ne concernent pas directement la lutte contre le changement climatique, peuvent être lus en parallèle, mais bizarrement (souci de maîtrise de la langue française peut-être ?), Malm ne les mentionne pas. Sans viser à l'exhaustivité, ils recensent divers moyens de combattre une oppression, montrant, non leur opposition, mais leur complémentarité. La question du pouvoir incite à dépasser un élément aujourd'hui de principe, quasi religieux, brandi par une organisation comme Extinction Rebellion - XR, contre lequel s'insurge Malm. Dans le même ordre d'idées, rappelons le "paradoxe de la tolérance " énoncé en son temps par Karl Popper: une société tolérante doit-elle tolérer l'intolérance? Aussi paradoxal que cela paraisse, répond le philosophe autrichien, défendre la tolérance nécessite de ne pas tolérer l'intolérance. C'était, il y a bien longtemps, le pourquoi de la création d'une revue appelée Article 31: défendre la démocratie en dénonçant les ennemis de cette démocratie (savoir si la démocratie est le meilleur système politique relève d'un autre débat), en l'occurrence, ici, l'extrême droite. Il en va de même avec le respect de la nature. La non-violence nécessite l'emploi éventuel de la violence pour pouvoir être affirmée. L'apparent paradoxe n'est qu'une règle de survie. C'est ce qu'Andreas Malm vient rappeler fort à propos, en évoquant d'une part le mouvement du Général Ludd (on ne sait si ce personnage a réellement existé, mais il a fait des émules) de la fin du XVIII et du début du XIX en GrandeBretagne, et appelant à des actions de sabotage ciblées et massives, et d'autre part, le mouvement des suffragettes, en Grande-Bretagne également, au tournant des XIX et $\mathrm{XX}^{\mathrm{e}}$ siècles. Il ne s'agit alors pas de "terrorisme », observe-t-il, qui frappe aveuglément et ne s'en prend pas qu'aux biens, mais de "vandalisme», ou, plus justement, de "sabotage", mot que le mouvement ouvrier s'était assez brièvement approprié (pensons à Pouget) avant, hélas, de le juger offensant. Les écologistes officiels, élus ou souhaitant l'être, devraient se féliciter, note Malm, non sans une certaine perfidie, de "l'influence bénéfique d'un flanc radical»- en s'empressant de le dénoncer, bien entendu, pour rester crédibles face à leurs interlocuteurs, les gens de pouvoir. Distinction sémantique d'une importance extrême, car les nantis (pollueurs, militaires, actionnaires, chefs de tout ordre) ont vite fait d'imposer à leurs ennemis des étiquettes infamantes afin de les réduire plus facilement au silence.

6 Nos quelques réserves énoncées doivent donc surtout donner à penser combien, ce livre est un outil pour ne plus stagner dans la lutte écologique. « ...La résistance est la voie de la survie par tous les temps ", conclut Andreas Malm. À lire avec l'excellent roman Le Zoo de Mengele du Norvégien Gert Nygårdshaug (paru il y a quelques années, mais plus que jamais d'actualité !), avant, enfin, de passer aux travaux pratiques... 


\section{NOTES}

1. Cet argument est par ailleurs souligné par Karin Bojs, concitoyenne d'Andreas Malm, dans Ma grande famille, Les Arènes, 2020, un riche essai sur les étapes du peuplement de l'Europe. 